\title{
THREE SOLUTIONS FOR A PERTURBED SUBLINEAR ELLIPTIC PROBLEM IN $\mathbb{R}^{N}$
}

\author{
GIOVANNI ANELLO and GIUSEPPE CORDARO \\ Department of Mathematics, University of Messina, 98166 Sant'Agata-Messina, Italy \\ e-mail: anello@dipmat.unime.it; cordaro@dipmat.unime.it
}

(Received 3 August, 2004; accepted 4 October, 2004)

\begin{abstract}
In this paper we study a perturbed sublinear elliptic problem in $\mathbb{R}^{N}$. In particular, using variational methods, we establish a result that ensures the existence of at least three weak solutions.
\end{abstract}

2000 Mathematics Subject Classification. 35J20.

1. Introduction. In this paper we are concerned with the following perturbation problem

$$
\left\{\begin{array}{l}
-\Delta u=h(x)|u|^{s-2} u+\lambda f(x, u) \text { in } \mathbb{R}^{N} \\
u \in D^{1,2}\left(\mathbb{R}^{N}\right)
\end{array}\right.
$$

where $s \in] 1,2\left[, N \geq 3, \lambda \in \mathbb{R}_{+}, f: \mathbb{R}^{N} \times \mathbb{R} \rightarrow \mathbb{R}\right.$ is a Carathéodory function, $h \in$ $L_{l o c}^{\infty}\left(\mathbb{R}^{N}\right) \cap L^{\frac{2 N}{2 N-s(N-2)}}\left(\mathbb{R}^{N}\right)$ is a function almost everywhere nonnegative in $\mathbb{R}^{N}$ such that, for some set $A \subseteq \mathbb{R}^{N}$ of positive measure, ess $\inf _{A} h>0$ and

$$
D^{1,2}\left(\mathbb{R}^{N}\right)=\left\{u \in L^{\frac{2 N}{N-2}}\left(\mathbb{R}^{N}\right) / \nabla u \in L^{2}\left(\mathbb{R}^{N}\right)\right\}
$$

is the completion of

$$
C_{0}\left(\mathbb{R}^{N}\right)=\left\{u \in C\left(\mathbb{R}^{N}\right): \operatorname{supp}(u)(\text { support of } u) \text { is compact }\right\}
$$

with respect to the norm

$$
\|u\|=\left(\int_{\mathbb{R}^{N}}|\nabla u|^{2} d x\right)^{\frac{1}{2}}
$$

induced by the scalar product

$$
(u, v)=\int_{\mathbb{R}^{N}} \nabla u(x) \nabla v(x) d x .
$$

Moreover, we assume that the function $f$ satisfies the following conditions.

(a) There exist $q \in\left[0, \frac{N+2}{N-2}\left[, \alpha \in L^{t}\left(\mathbb{R}^{N}\right) \cap L_{\text {loc }}^{\infty}\left(\mathbb{R}^{N}\right)\right.\right.$, where we set $t=\frac{2 N}{N+2-q(N-2)}$, $\beta \in L^{\frac{2 N}{N+2}}\left(\mathbb{R}^{N}\right) \cap L_{\text {loc }}^{\infty}\left(\mathbb{R}^{N}\right)$ such that

$$
|f(x, t)| \leq \alpha(x)|t|^{q}+\beta(x),
$$

for a.e. $x \in \mathbb{R}^{N}$ and all $t \in \mathbb{R}$. 
(b) There exist three functions $\gamma \in L^{\frac{N}{2}}\left(\mathbb{R}^{N}, \mathbb{R}_{+}\right), \delta \in L^{\frac{2 N}{N+2}}\left(\mathbb{R}^{N}, \mathbb{R}_{+}\right)$and $\chi \in$ $L^{1}\left(\mathbb{R}^{N}, \mathbb{R}_{+}\right)$such that

$$
\int_{0}^{t} f(x, s) d s \leq \gamma(x)|t|^{2}+\delta(x)|t|+\chi(x),
$$

for a.e. $x \in \mathbb{R}^{N}$ and all $t \in \mathbb{R}$.

For each $u \in D^{1,2}\left(\mathbb{R}^{N}\right)$,

$$
E(u)=\frac{1}{2} \int_{\mathbb{R}^{N}}|\nabla u(x)|^{2} d x-\frac{1}{s} \int_{\mathbb{R}^{N}} h(x)|u(x)|^{s}-\lambda \int_{\mathbb{R}^{N}}\left(\int_{0}^{u(x)} f(x, s) d s\right) d x
$$

is the energy functional relative to problem $\left(P_{\lambda}\right)$. In proving our result, we will show that the above assumptions on $h$ and $f$ ensure, in particular, that $E$ is continuously differentiable on $D^{1,2}\left(\mathbb{R}^{N}\right)$. Hence the weak solutions of $\left(P_{\lambda}\right)$ corresponds to the critical points of $E$.

Our result is as follows.

THEOREM 1.1. Under the assumptions stated above there exist $\sigma, \bar{\lambda}>0$ such that, for each $\lambda \in\left[0, \bar{\lambda}\left[\right.\right.$, problem $\left(P_{\lambda}\right)$ admits at least three distinct solutions in $D^{1,2}\left(\mathbb{R}^{N}\right)$ whose norms are not greater than $\sigma$.

In the literature, most of the papers that deal with multiple solutions for elliptic equations on $\mathbb{R}^{N}$ consider particular types of nonlinearities as power functions, convex or concave functions; see for instance [4, 9]. This is due to the lack of compact embeddings for Sobolev spaces on unbounded domains which, in many cases, represents the main difficulty in using a variational approach. Such a difficulty is often overcome by exploiting the particular properties of the nonlinearity as homogeneities or symmetries.

In this paper, we consider a nonlinearity which is a pertubation of a particular symmetric sublinear nonlinearity (i.e. $h(x)|t|^{s-2} t$ ) by a function $f$ that must satisfy only the growth conditions (a) and (b). This kind of nonlinearity allows us to study our problem by means of variational methods and, in particular, to apply a consequence (Theorem 2.1 in [1]) of a variational principle stated in [6].

The paper is organized into three sections. In Section 2 some preliminary results are stated and proved. Section 3 contains the proof of Theorem 1.1.

2. Preliminary lemmas. For the reader's convenience, we begin this section with the following known result.

LEMMA 2.1. Let $\left\{u_{n}\right\}_{n \in \mathbb{N}}$ be a sequence weakly converging to $u$ in $D^{1,2}\left(\mathbb{R}^{N}\right)$. Then, for $p \in\left[1, \frac{2 N}{N-2}\left[\right.\right.$ and for any bounded measurable set $\Omega \subseteq \mathbb{R}^{N},\left\{u_{n \mid \Omega}\right\}_{n \in \mathbb{N}}$ strongly converges to $u_{\mid \Omega}$ in $L^{p}(\Omega)$.

Proof. Consider $\left\{u_{n}\right\}_{n \in \mathbb{N}}$ weakly converging to $u$ in $D^{1,2}\left(\mathbb{R}^{N}\right)$. We note that $u_{n} \rightarrow u$ weakly in $L^{\frac{2 N}{N-2}}\left(\mathbf{R}^{N}\right)$. Hence, in particular, for every $v \in C_{0}^{\infty}\left(\mathbb{R}^{N}\right)$, one has

$$
\lim _{n \rightarrow \infty} \int_{\mathbb{R}^{N}} u_{n}(x) v(x) d x=\int_{\mathbf{R}^{N}} u(x) v(x) d x .
$$


Fix $R>0, p \in\left[1, \frac{2 N}{N-2}\right.$ [ and set $B_{R}=\left\{x \in \mathbf{R}^{N}:|x| \leq R\right\}$. We prove that $u_{n} \rightarrow u$ strongly in $L^{p}\left(B_{R}\right)$.

Indeed, denote by $u_{\mid B_{R}}$ the restriction of $u$ to $B_{R}$ and suppose that $\left\{u_{n}\right\}$ does not converge to $u_{\mid B_{R}}$ weakly in $W^{1,2}\left(B_{R}\right)$. Since $\left\{u_{n}\right\}$ is norm bounded in $W^{1,2}\left(B_{R}\right)$, there exist a subsequence $\left\{u_{n_{k}}\right\}$ and $\bar{u} \in W^{1,2}\left(B_{R}\right)$, with $\bar{u} \neq u_{\mid B_{R}}$, such that $u_{n_{k}} \rightarrow \bar{u}$ weakly in $W^{1,2}\left(B_{R}\right)$. By the Rellich-Kondrachov's Theorem, $u_{n_{k}} \rightarrow \bar{u}$ strongly in $L^{p}\left(B_{R}\right)$.

Taking into account (2.1), we obtain

$$
\int_{B_{R}} u(x) v(x) d x=\lim _{k \rightarrow \infty} \int_{B_{R}} u_{n_{k}}(x) v(x) d x=\int_{B_{R}} \bar{u}(x) v(x) d x,
$$

for every $v \in C_{0}^{\infty}\left(B_{R}\right)$. Then $u(x)=\bar{u}(x)$, for almost all $x \in B_{R}$, against the fact that $\bar{u} \neq u_{\mid B_{R}}$. Hence $\left\{u_{n}\right\}$ weakly converges to $u_{\mid B_{R}}$ in $W^{1,2}\left(B_{R}\right)$. Applying the RellichKondrachov's Theorem again, we conclude that $\left\{u_{n}\right\}$ strongly converges to $u$ in $L^{p}\left(B_{R}\right)$.

LEMMA 2.2. Condition (a) implies that the functional

$$
\Phi: u \rightarrow \int_{\mathbb{R}^{N}}\left(\int_{0}^{u(x)} f(x, t) d t\right) d x
$$

is well-defined, strongly continuous and Gateâux differentiable in $D^{1,2}\left(\mathbb{R}^{N}\right)$ with compact Gateâux derivative.

Proof. By standard arguments, $\Phi$ is proved to be well-defined, strongly continuous and Gateâux differentiable in $D^{1,2}\left(\mathbb{R}^{N}\right)$ and so we limit ourselves to prove that the Gateâux derivative $\Phi^{\prime}$ is compact.

Let $\left\{u_{n}\right\}$ be a bounded sequence in $D^{1,2}\left(\mathbb{R}^{N}\right)$. There exist $\bar{u} \in D^{1,2}\left(\mathbb{R}^{N}\right)$ and a subsequence, which we denote by $\left\{u_{n}\right\}$ again, weakly converging to $\bar{u}$ in $D^{1,2}\left(\mathbb{R}^{N}\right)$. Now, fix $\epsilon>0$ arbitrarily and choose $M>0$ such that

$$
\|\alpha\|_{L^{t}(|x|>M)}<\epsilon,\|\beta\|_{L^{\frac{2 N}{N+2}(|x|>M)}}<\epsilon .
$$

For every $v \in H$ with $\|v\|=1$ and $n \in \mathbb{N}$, one has

$$
\begin{aligned}
\left|\left(\Phi\left(u_{n}\right)-\Phi(\bar{u})\right)(v)\right| \leq & \left|\int_{|x| \leq M}\left(f\left(x, u_{n}(x)\right)-f(x, \bar{u}(x))\right) v(x) d x\right| \\
& \left.+\mid \int_{|x|>M} f\left(x, u_{n}(x)\right)-f(x, \bar{u}(x))\right) v(x) d x \mid \\
& \leq C_{1}\left(\int_{|x| \leq M}\left|f\left(x, u_{n}(x)\right)-f(x, \bar{u}(x))\right|^{\frac{2 N}{N+2}}\right)^{\frac{N+2}{2 N}} \\
& +C_{2}\|\alpha\|_{L^{t}(|x|>M)}^{\frac{N+2}{2 N}}+C_{3}\|\beta\|_{L^{\frac{2 N}{N+2}}(|x|>M)},
\end{aligned}
$$

where $C_{1}, C_{2}, C_{3}$ are suitable positive constants. By Lemma 2.1, $\left\{u_{n}\right\}$ converges strongly in $L^{q+1}(|x| \leq M)$, so that $f\left(\cdot, u_{n}(\cdot)\right) \rightarrow f(\cdot, \bar{u}(\cdot))$ strongly in $L^{\frac{2 N}{N+2}}(|x| \leq M)$. Hence, there exists $v \in \mathbb{N}$ such that, for $n>v$, one has

$$
\left\|\Phi\left(u_{n}\right)-\Phi(\bar{u})\right\|_{H^{*}}=\sup _{v \in H,\|v\|=1}\left|\left(\Phi\left(u_{n}\right)-\Phi(\bar{u})\right)(v)\right| \leq C_{1} \epsilon+C_{2} \epsilon^{\frac{N+2}{2 N}}+C_{3} \epsilon .
$$

This proves that $\Phi^{\prime}$ is a compact operator. 
Lemma 2.3. Let $\Psi: D^{1,2}\left(\mathbb{R}^{N}\right) \rightarrow \mathbb{R}$ be defined by

$$
\Psi(u)=\frac{1}{2}\|u\|^{2}-\frac{1}{s} \int_{\mathbb{R}^{N}} h(x)|u(x)|^{s} d x .
$$

There exists a unique $u_{0} \in D^{1,2}\left(\mathbb{R}^{N}\right) \cap C\left(\mathbb{R}^{N}\right)$, with $u_{0}>0$ in $\mathbb{R}^{N}$, such that

$$
\Psi\left(u_{0}\right)=\inf _{u \in D^{1,2}\left(\mathbb{R}^{N}\right)} \Psi(u) .
$$

Proof. By Lemma 2.2, the functional

$$
u \rightarrow \int_{\mathbb{R}^{N}} h(x)|u(x)|^{s} d x
$$

has compact Gateâux derivative in $D^{1,2}\left(\mathbb{R}^{N}\right)$. Hence, in particular, it follows that $\Psi$ is weakly sequentially lower semicontinuous. Since $\Psi$ is also coercive, $\Psi(u)=\Psi(|u|)$ and $\inf _{D^{1,2}\left(\mathbb{R}^{N}\right)} \Psi<0$, there exists $u_{0} \in D^{1,2}\left(\mathbb{R}^{N}\right) \backslash\{0\}$ such that

$$
\Psi\left(u_{0}\right)=\inf _{D^{1,2}\left(\mathbb{R}^{N}\right)} \Psi,
$$

with $u_{0} \geq 0$ almost everywhere in $\mathbb{R}^{N}$. Consequently $u_{0}$ is a weak solution of the problem

$$
\left\{\begin{array}{l}
-\Delta u=h(x)|u|^{s-2} u \text { in } \mathbb{R}^{N}, \\
u \in D^{1,2}\left(\mathbb{R}^{N}\right) .
\end{array}\right.
$$

From Lemma B.3 in [8] and Theorem 8.22 in [3], it follows that $u_{0} \in C\left(\mathbb{R}^{N}\right)$. Moreover the strong maximum principle ensures that $u_{0}(x)>0$, for all $x \in \mathbb{R}^{N}$.

Now, for each $R>0$, set $B_{R}=\left\{x \in \mathbb{R}^{N}:|x|<R\right\}$ and let $u_{R} \in W_{0}^{1,2}\left(B_{R}\right)$ such that

$$
\Psi\left(u_{R}\right)=\inf _{u \in W_{0}^{1,2}\left(B_{R}\right)} \Psi(u),
$$

and $u_{R} \geq 0$ a.e. in $B_{R}$. The function $u_{R}$ exists and is unique. Also the net $\left\{u_{R}\right\}_{R>0}$ is increasing with respect to $R$; see [2]. Moreover, by definition, the net $\left\{\Psi\left(u_{R}\right)\right\}$ is not increasing with respect to $R$ and is bounded below by $\inf _{D^{1,2}\left(\mathbb{R}^{N}\right)} \Psi$. We deduce that

$$
\lim _{R \rightarrow+\infty} \Psi\left(u_{R}\right) \geq \inf _{u \in D^{1,2}\left(\mathbb{R}^{N}\right)} \Psi(u) .
$$

Since $\Psi$ is coercive, there exist a subsequence $\left\{R_{n}\right\}$, with $\lim _{n \rightarrow \infty} R_{n}=+\infty$, and $\tilde{u} \in$ $D^{1,2}\left(\mathbb{R}^{N}\right)$ such that $u_{R_{n}} \rightarrow \tilde{u}$ weakly in $D^{1,2}\left(\mathbb{R}^{N}\right)$ as $n \rightarrow \infty$. Hence, we exploit the sequentially weak lower semicontinuity of $\Psi$ and obtain

$$
\Psi(\tilde{u}) \leq \lim _{n \rightarrow \infty} \Psi\left(u_{R_{n}}\right)=\lim _{R \rightarrow+\infty} \Psi\left(u_{R}\right) .
$$

Furthermore, by Lemma 2.1 and the monotony of $\left\{u_{R}\right\}_{R>0}$, we also have that

$$
\lim _{R \rightarrow+\infty} u_{R}(x)=\lim _{n \rightarrow \infty} u_{R_{n}}(x)=\tilde{u}(x),
$$

for a.e. $x \in \mathbb{R}^{N}$. 
We claim that $\Psi(\tilde{u})=\inf _{D^{1,2}\left(\mathbb{R}^{N}\right)} \Psi$. Suppose that

$$
\Psi(\tilde{u})>\inf _{D^{1,2}\left(\mathbb{R}^{N}\right)} \Psi .
$$

Consequently, there would exist $v \in C_{0}^{\infty}\left(\mathbb{R}^{N}\right)$ such that

$$
\Psi(v)<\Psi(\tilde{u}) .
$$

On the other hand, if we choose $\bar{R}>0$ such that $\operatorname{supp}(v) \subseteq B_{\bar{R}}$, we also have

$$
\Psi(v) \geq \inf _{u \in W_{0}^{1,2}\left(B_{\bar{R}}\right)} \Psi(u)=\Psi\left(u_{\bar{R}}\right) \geq \Psi(\tilde{u}),
$$

contradicting (2.4).

Let $\bar{u} \in D^{1,2}\left(\mathbb{R}^{N}\right)$ be such that

$$
\Psi(\bar{u})=\inf _{D^{1,2}\left(\mathbb{R}^{N}\right)} \Psi,
$$

with $\bar{u} \geq 0$ a.e. in $\mathbb{R}^{N}$. It turns out that $\bar{u}=\tilde{u}$.

Indeed, for each $R>0, \bar{u}$ is a supersolution for the problem

$$
\begin{cases}-\Delta u=h(x) u^{s-1} & \text { in } B_{R} \\ u=0 & \text { on } \partial B_{R}\end{cases}
$$

so that $u_{R} \leq \bar{u}$ a.e. in $\mathbb{R}^{N}$. Passing to the limit as $R \rightarrow+\infty$, we find that

$$
\tilde{u}(x) \leq \bar{u}(x)
$$

for a.e. $x \in \mathbb{R}^{N}$. From the fact that $\tilde{u}$ and $\bar{u}$ are, in particular, critical points of $\Psi$ such that $\Psi(\tilde{u})=\Psi(\bar{u})$, it follows that

$$
\int_{\mathbb{R}^{N}} h(x)\left[(\tilde{u}(x))^{s}-(\bar{u}(x))^{s}\right] d x=0 .
$$

Consequently, from (2.5) and (2.6), $\tilde{u}(x)=\bar{u}(x)$ for a.e. $x \in \mathbb{R}^{N}$.

LeMma 2.4. Let $\Psi$ and $u_{0}$ be as in Lemma 2.3. For every $u \in D^{1,2}\left(\mathbb{R}^{N}\right)$, the real function

$$
r \rightarrow \inf _{\|w\|=r} \Psi(u+w)
$$

is continuous in $\mathbb{R}_{+}$. Moreover, there exists $r_{0}>0$ such that, for every $0<r<r_{0}$, we have

$$
\Psi\left( \pm u_{0}\right)<\inf _{\|v\|=r} \Psi\left(v \pm u_{0}\right)
$$

Proof. Fix $u \in D^{1,2}\left(\mathbb{R}^{N}\right)$. For each $r>0$, one has

$$
\inf _{\|v\|=r} \Psi(u+v)=\frac{1}{2} r^{2}+\frac{1}{2}\|u\|^{2}-\sup _{\|v\|=r}\left((u, v)-\frac{1}{s} \int_{\mathbb{R}^{N}} h(x)|u(x)+v(x)|^{s} d x\right) .
$$


Hence the continuity follows from the weak sequential continuity of the function

$$
v \rightarrow(u, v)-\frac{1}{s} \int_{\mathbb{R}^{N}} h(x)|u(x)+v(x)|^{s} d x .
$$

See Lemma 6 of [7].

Let $r_{0}=2\left\|u_{0}\right\|$ and suppose that, for some $0<\bar{r}<r_{0}$, we have

$$
\Psi\left(u_{0}\right)=\inf _{\|v\|=\bar{r}} \Psi\left(u_{0}+v\right) .
$$

Then, we can find a sequence $\left\{u_{k}\right\}$ in $D^{1,2}\left(\mathbb{R}^{N}\right)$ with $\left\|u_{k}\right\|=\bar{r}$, for all $k \in \mathbb{N}$, such that

$$
\lim _{k \rightarrow+\infty} \Psi\left(u_{0}+u_{k}\right)=\Psi\left(u_{0}\right)
$$

Up to a subsequence, we can suppose that $u_{k} \rightarrow \bar{u}$ weakly in $H$. In particular, we have $\|\bar{u}\| \leq \bar{r}$,

$$
\left(u_{k}, u_{0}\right)=\int_{\Omega} \nabla u_{k}(x) \nabla u_{0}(x) d x \rightarrow \int_{\mathbb{R}^{N}} \nabla \bar{u}(x) \nabla u_{0}(x) d x=\left(\bar{u}, u_{0}\right)
$$

and

$$
\int_{\mathbb{R}^{N}} h(x)\left|u_{k}(x)+u_{0}(x)\right|^{s} d x \rightarrow \int_{\mathbb{R}^{N}} h(x)\left|\bar{u}(x)+u_{0}(x)\right|^{s} d x,
$$

as $k \rightarrow \infty$.

Consequently, from (2.2), (2.7), (2.8) and (2.9), one has

$$
\begin{aligned}
\inf _{D^{1,2}\left(\mathbb{R}^{N}\right)} \Psi & =\Psi\left(u_{0}\right)=\lim _{k \rightarrow+\infty} \Psi\left(u_{0}+u_{k}\right) \\
& =\lim _{k \rightarrow+\infty}\left(\frac{1}{2}\left\|u_{0}\right\|^{2}+\frac{1}{2} \bar{r}^{2}+\left(u_{k}, u_{0}\right)-\frac{1}{s} \int_{\mathbb{R}^{N}} h(x)\left|u_{k}(x)+u_{0}(x)\right|^{s} d x\right) \\
& =\frac{1}{2}\left\|u_{0}\right\|^{2}+\frac{1}{2} \bar{r}^{2}+\left(\bar{u}, u_{0}\right)-\frac{1}{s} \int_{\mathbb{R}^{N}} h(x)\left|\bar{u}(x)+u_{0}(x)\right|^{s} d x \\
& \geq \frac{1}{2}\left\|u_{0}\right\|^{2}+\frac{1}{2}\|\bar{u}\|^{2}+\left(\bar{u}, u_{0}\right)-\frac{1}{s} \int_{\mathbb{R}^{N}} h(x)\left|\bar{u}(x)+u_{0}(x)\right|^{s} d x=\Psi\left(\bar{u}+u_{0}\right) .
\end{aligned}
$$

From this we deduce that $\bar{u}+u_{0}$ as well as $\left|\bar{u}+u_{0}\right|$ are global minima for $\Psi$ and that $\|\bar{u}\|=\bar{r}$. Using the same arguments to prove regularity and positivity of $u_{0}$, we have $\left|\bar{u}+u_{0}\right|, \bar{u}+u_{0} \in C\left(\mathbb{R}^{N}\right)$ and $\left|\bar{u}(x)+u_{0}(x)\right|>0$, for all $x \in \mathbb{R}^{N}$. Note that $\bar{u} \in C\left(\mathbb{R}^{N}\right)$, being $\bar{u}=\left(\bar{u}+u_{0}\right)-u_{0}$. By applying Lemma 2.3 , we obtain

$$
\left|\bar{u}(x)+u_{0}(x)\right|=u_{0}(x)
$$

for all $x \in \mathbb{R}^{N}$. At this point, put

$$
A=\left\{x \in \mathbb{R}^{N}: \bar{u}(x)=0\right\}
$$

and

$$
B=\left\{x \in \mathbb{R}^{N}: \bar{u}(x)=-2 u_{0}(x)\right\} .
$$


By (2.10), $A \cup B=\mathbb{R}^{N}$ and, because $u_{0}>0$ in $\mathbb{R}^{N}, A \cap B=\emptyset$. Moreover, the continuity of $\bar{u}, u_{0}$, implies that $A$ and $B$ are closed sets in $\mathbb{R}^{N}$. Since $\mathbb{R}^{N}$ is connected, it results that $A=\mathbb{R}^{N}$ or $B=\mathbb{R}^{N}$.

In the former case, it should be $\bar{u}=0$ against the fact that $\|\bar{u}\|=\bar{r}>0$. In the latter case, it should be $\bar{u}=-2 u_{0}$ and hence $\|\bar{u}\|=2\left\|u_{0}\right\|$ contrary to $\bar{r}<2\left\|u_{0}\right\|$.

Taking into account that $\Psi\left(-u_{0}\right)=\Psi\left(u_{0}\right)$, the same conclusion holds with $-u_{0}$ in place of $u_{0}$, as can be easily checked.

3. Proof of Theorem 1.1. Consider the functionals $\Psi, \Phi: D^{1,2}\left(\mathbb{R}^{N}\right) \rightarrow \mathbb{R}$ defined as follows:

$$
\begin{aligned}
& \Psi(u)=\frac{1}{2}\|u\|^{2}-\frac{1}{s} \int_{\mathbb{R}^{N}} h(x)|u(x)|^{s} d x, \\
& \Phi(u)=-\int_{\mathbb{R}^{N}}\left(\int_{0}^{u(x)} f(x, t) d t\right) d x .
\end{aligned}
$$

By Lemma 2.2, it follows that $\Phi$ is weakly sequentially continuous and $\Psi$ is weakly sequentially lower semicontinuous in $D^{1,2}\left(\mathbb{R}^{N}\right)$. By condition (b), it also follows that there exists $\lambda_{1}>0$ such that, for every $\lambda \in\left[0, \lambda_{1}[\right.$, the functional $\Psi+\lambda \Phi$ is coercive.

Put $x_{1}=u_{0}, x_{2}=-u_{0}$ and fix $0<\bar{r}<\min \left\{\left\|u_{0}\right\|, r_{0}\right\}$. By Lemma 2.4 it follows that the hypotheses of Theorem 2.1 in [1] are satisfied and so there exists $0<\bar{\lambda}<\lambda_{1}$ such that, for every $\lambda \in] 0, \bar{\lambda}\left[, \Psi+\lambda \Phi\right.$ admits two distinct local minima $u_{1}^{(\lambda)}, u_{2}^{(\lambda)} \in H$, with $\left\|x_{i}-u_{i}^{(\lambda)}\right\|<\bar{r}$, for $i=1,2$. Fix $\lambda \in[0, \bar{\lambda}[$. Since $\Psi+\lambda \Phi$ satisfies the Palais-Smale condition (see Example 38.25 in [10]), Theorem 1 of [5] implies the existence of a third critical point $u_{3}^{(\lambda)}$, distinct from $u_{1}^{(\lambda)}$ and $u_{2}^{(\lambda)}$, that satisfies

$$
\Psi\left(u_{3}^{(\lambda)}\right)+\lambda \Phi\left(u_{3}^{(\lambda)}\right)=\phi(\lambda)
$$

where

$$
\phi(\lambda)=\inf _{\psi \in \Gamma_{\lambda}} \sup _{t \in[0,1]}(\Psi(\psi(t))+\lambda \Phi(\psi(t)))
$$

and

$$
\Gamma_{\lambda}=\left\{\psi \in C([0,1], H): \psi(0)=u_{1}^{(\lambda)} \text { and } \psi(1)=u_{2}^{(\lambda)}\right\}
$$

We note that, for every $\lambda \in\left[0, \bar{\lambda}\left[\right.\right.$, the function $\psi_{\lambda}: t \in[0,1] \rightarrow u_{1}^{(\lambda)}+(1-t) u_{2}^{(\lambda)}$ belongs to $\Gamma_{\lambda}$. Moreover, it follows that

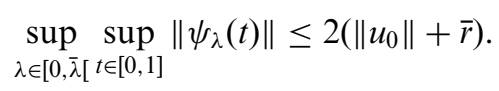

Exploiting the fact that $\Psi$ is the sum of the norm squared and a sequentially weakly continuous functional and that $\Phi$ is sequentially weakly continuous, one has

$$
\begin{aligned}
\sup _{\lambda \in[0, \bar{\lambda}[} \phi(\lambda) & \leq \sup _{\lambda \in[0, \bar{\lambda}[t \in[0,1]} \sup _{t \in[}\left(\Psi\left(\psi_{\lambda}(t)\right)+\lambda \Phi\left(\psi_{\lambda}(t)\right)\right) \\
& \leq \sup _{\|v\| \leq 2\left(\left\|u_{0}\right\|+\bar{r}\right)} \Psi(v)+\bar{\lambda} \sup _{\|v\| \leq 2\left(\left\|u_{0}\right\|+\bar{r}\right)} \Phi(v)<+\infty
\end{aligned}
$$


At this point, we can prove that $\sup _{\lambda \in[0, \bar{\lambda}[}\left\|u_{3}^{(\lambda)}\right\|<\infty$. In fact, if

$$
\sup _{\lambda \in[0, \bar{\lambda}[}\left\|u_{3}^{(\lambda)}\right\|=+\infty
$$

there would exist a bounded sequence $\left\{\lambda_{n}\right\} \subset\left[0, \bar{\lambda}\left[\right.\right.$ such that $\left\|u_{3}^{\left(\lambda_{n}\right)}\right\| \rightarrow+\infty$, as $n \rightarrow \infty$. Consequently, we should have

$$
\begin{aligned}
\lim _{n \rightarrow \infty} \phi\left(\lambda_{n}\right) & =\lim _{n \rightarrow \infty}\left(\Psi\left(u_{3}^{\left(\lambda_{n}\right)}\right)+\lambda_{n} \Phi\left(u_{3}^{\left(\lambda_{n}\right)}\right)\right) \\
& \geq \lim _{n \rightarrow \infty}\left(\Psi\left(u_{3}^{\left(\lambda_{n}\right)}\right)+\bar{\lambda} \min \left\{0, \Phi\left(u_{3}^{\left(\lambda_{n}\right)}\right)\right\}\right)=+\infty
\end{aligned}
$$

contradicting (3.11).

Hence, set

$$
\sigma=\max \left\{2\left(\left\|u_{0}\right\|+\bar{r}\right), \sup _{\lambda \in[0, \bar{\lambda}[}\left\|u_{3}^{(\lambda)}\right\|\right\} .
$$

The conclusion follows.

\section{REFERENCES}

1. G. Anello, A multiplicity theorem for critical points of functionals on reflexive Banach spaces, Arch. Math. (Basel) 82 (2004), 172-179.

2. H. Brezis and S. Kamin, Sublinear elliptic equations in $\mathbb{R}^{N}$, Manuscripta Math. $\mathbf{7 4}$ (1992), 87-106.

3. D. Gilbarg and N. S. Trudinger, Elliptic partial differential equations of second order (Springer-Verlag, 1977).

4. A. Malchiodi, Multiple positive solutions of some elliptic equations on $\mathbb{R}^{N}$, Nonlinear Anal. 43 (2001), 159-172. $142-149$

5. P. Pucci and J. Serrin, A mountain pass theorem, J. Differential Equations 60 (1985),

6. B. Ricceri, A general variational principle and some of its applications, J. Comput. Appl. Math. 113 (2000), 401-410.

7. M. Schechter and K. Tintarev, Spherical maxima in Hilbert space and semilinear elliptic eigenvalue problems, Differential Integral Equations 3 (5) (1990), 889-899.

8. M. Struwe, Variational methods (Springer-Verlag, 1996).

9. S. B. Tshinanga, On multiple solutions of semilinear elliptic equation on unbounded domains with concave and convex nonlinearities, Nonlinear Anal. 28 (1997), 809-814. 1984).

10. E. Zeidler, Nonlinear functional analysis and its applications, Vol. III (Springer-Verlag, 